\title{
Optical Night-Sky Brightness at Mauna Kea over the Course of a Complete Sunspot Cycle
}

\author{
K. Krisciunas ${ }^{1}$ \\ Department of Astronomy, University of Washington, Box 351580, Seattle, WA 98195 \\ Electronic mail: kevin@astro.washington.edu
}

Received —

\footnotetext{
${ }^{1}$ Based on data obtained when the author was at the Joint Astronomy Centre, Hilo, Hawaii.
} 


\begin{abstract}
We have produced a data base of $V$-band and $B$-band night-sky brightness measurements rather evenly spread out over the course of a whole sunspot cycle from September 1985 to August 1996. Almost all the data were obtained at the 2800-m level of Mauna Kea using the same telescope, same photomultiplier tube, filters, and diaphragm, thus minimizing various sources of systematic error and allowing an estimate of the sources of random error. The yearly $V$-band averages of observed sky brightness ranged from 21.287 to 21.906 magnitudes per square arc second. The color of the sky is $B-V=0.930$ and does not change discernibly over the course of the sunspot cycle. After correcting the $V$-band data to the zenith, we find that the airglow component varied a factor of 4.5 over the course of the solar cycle. Once the 11-year solar cycle effect is removed from the data, the most significant contribution to the scatter of individual data points appears to be the short term variations on time scales of tens of minutes like those observed by the Whole Earth Telescope project.
\end{abstract}

Subject headings: night-sky brightness 


\section{Introduction}

One of the most impressive sights in nature is the nighttime sky unmarred by artificial light and with only a thin crescent Moon or no Moon in the sky. On the Island of Hawaii the air is often transparent enough that if one is fully dark adapted, the starlight alone can cast a shadow of one's hand on one's chest, if one is wearing a light colored coat. This holds for clear, moonless nights at sea level, at the top of Mauna Kea, or at elevations in between. P

When doing astronomical photometry one wishes to determine the brightness of an individual object such as a star. One measures the star plus a certain amount of surrounding sky, then must measure blank sky nearby to obtain the light due to the star only. Even in the absence of sources of artificial light scattered into the beam, the "blank" sky is not completely dark owing to four sources of light (Roach \& Gordon 1973): (1) zodiacal light and the gegenschein (caused by sunlight scattered off interplanetary dust); (2) faint unresolved stars and atomic processes within our Galaxy; (3) diffuse extragalactic light (due to distant, faint unresolved galaxies); and (4) airglow and aurorae (produced by photochemical reactions in the Earth's upper atmosphere).

Roach \& Gordon (1973, p. 54) list some of the reactions giving rise to airglow. For example, continuum radiation from 500 to $650 \mathrm{~nm}$ wavelength is emitted when $\mathrm{NO}_{2}$ is produced from $\mathrm{O}$ and NO. This light would be observable in the Johnson $V$ - and $B$-bands. An equally strong source of emission is the line at $557.7 \mathrm{~nm}$, due to [O I]. This occurs in the middle of the Johnson $V$-band. It has been known for most of this century that the airglow varies over the course of the solar cycle. (See Walker 1988 for further information

\footnotetext{
${ }^{2}$ While this fact is impressive, it is not that strange if one considers that the starlight from the whole sky amounts to 1160 stars of first magnitude (Allen 1973, p. 245).
} 
and relevant references). If we think of the airglow as a low-level aurora, this makes sense. As the solar wind energizes the Earth's atmosphere during the day, detectable results can be observed at night.

The airglow not only varies on times scales of years, but it systematically gets fainter over the course of a given night (see Fig. 2 of Walker 1988). It can even vary measurably over time scales of minutes (see Fig. 4 of Pilachowski et al. 1989, Fig. 3 of Nather et al. 1990, Morrison et al. 1997, and Fig. 5 below). As a result it is not sensible to speak of the "intrinsic" night-sky level at even a well protected site, except in rather broad terms.

While my main purpose over the years in doing photometry was to study variable stars, it required very little extra effort to obtain the data presented here. The purpose of the present paper is to present further observations of the night-sky brightness at Mauna Kea (primarily measured at the $2800-\mathrm{m}$ level). We now have data that are roughly evenly distributed over a complete solar cycle. These data are important for long term studies of the quality of the site and for planning various observations. As we all know, the signal-to-noise ratio obtained while imaging astronomical objects depends on the contrast of the object itself and the sky background. It is not well known, however, that the optical sky brightness at a good site at solar maximum with no Moon in the sky is quite comparable to the sky brightness at solar minimum with a quarter Moon in the sky. (See Krisciunas \& Schaefer 1991 for a model of the brightness of moonlight.)

In this paper we shall also consider the systematic and random errors that enter night-sky measurements. 


\section{Observations}

The method of determining the night-sky brightness is laid out in Krisciunas (1990), hereafter referred to as Paper I, which contains data of 1985 through 1989. The principal system is described in Krisciunas (1996). The telescope is a 15-cm f/5.82 reflector. The photometer uses an uncooled RCA 931A photomultiplier tube, a DC amplifier, and a strip chart recorder. In short, a signal on the "blank" sky is made, with the strip chart recorder gain set so as to obtain sufficient resolution for digitizing the signal (typically 10 millivolts $\mathrm{cm}^{-1}$ for the $V$-band and 5 millivolts $\mathrm{cm}^{-1}$ for the $B$-band). One must also obtain dark current readings on the same strip chart gains. A nominal amplifier gain of $10^{6}$ was always used for the sky readings. One or more standard stars were observed near the zenith. Standard stars were measured with the strip chart recorder at a setting of 50 or 100 millivolts $\mathrm{cm}^{-1}$, and with amplifier gains on one of six settings between $10^{3.5}$ to $10^{6}$. Since one can determine a gain table for the amplifier in the lab and check for errors with observations of standards stars of different brightness, we feel that the gain settings are not a serious source of systematic error. Still, for the purposes of accurately measuring sky brightness, it is best to observe both the sky and appropriately faint standard stars on the same amplifier gain setting.

Let $\mathrm{D}_{s k y}$ be the net deflection on the sky (i.e. with the dark current subtracted off) and reduced to the same strip chart gain as the star measurement, $D_{\star}$ be the net deflection on a standard star (i.e. with sky and dark current subtracted off), $\Delta \mathrm{G}$ be the difference of amplifier gain (in magnitudes) between the readings on the sky and star, $\mathrm{k}_{\lambda}$ be the atmospheric extinction for the filter used (in magnitudes per air mass), $\mathrm{X}_{\star}$ be the air mass value of the standard star observed, and $\mathrm{M}_{\star}$ be the catalog magnitude of that standard star. Then the single-beam magnitude of the sky reading, made with reference to that standard star is: 


$$
S=-2.5 \log \left(\mathrm{D}_{\mathrm{sky}} / \mathrm{D}_{\star}\right)+\Delta \mathrm{G}+\mathrm{k}_{\lambda} \mathrm{X}_{\star}+\mathrm{M}_{\star}
$$

where the logarithm is to the base 10. If we let $\mathrm{A}$ be the area of the beam in square arc seconds, then the sky brightness in magnitudes per square arc second will be

$$
I(\mu)=S+2.5 \log \mathrm{A} .
$$

Throughout this paper we shall use the symbol $\mu$ to mean units of magnitudes per square arc second. $\mu_{V}$ and $\mu_{B}$ refer to sky brightness in magnitudes per square arc second in the $V$-band and $B$-band, respectively.

For our system the beam area is $6.522 \pm 0.184$ square arc minutes, and $2.5 \log \mathrm{A}$ is $10.927 \pm 0.031$. If two or three standard stars are used, one can then average the derived values. If one is carrying out all-sky photometry, in which one determines the extinction and transformation to the UBV system from observations of, say, a dozen standards, then one can derive the single beam night-sky magnitude by setting the air mass of the sky reading to zero. As stated in Paper I and described by Walker (1970), if the only component of the the sky brightness were faint background stars, one would treat it like an observation of any other star. But because airglow takes place in the atmosphere, not outside it, for sky brightness one must do something a bit different.

In Table 1 we give night-sky values from the years 1990 through 1996. The data of 9 February 1992 were obtained at the 4205-m Mauna Kea summit with the University of Hawaii 0.6-m telescope and an Optec SSP-5 photometer using a 23 arcsecond diameter beam. All the other data were taken with the system described above at the $2800-\mathrm{m}$ level of Mauna Kea. This is the mid-level facility at Hale Pohaku (Hawaiian for "house of stone"), the location of the Onizuka Visitors' Center. 
In Table $1 \mathrm{UT}_{V}$ is the Universal Time of the $V$-band measurement. The $B$-band measurement was taken within 5 minutes of this time. $\Delta \mathrm{T}_{t w i}$ is the time since the end of astronomical twilight (Sun elevation $-18^{\circ}$ ). $\alpha, \delta$, and Z, respectively, are the Right Ascension, Declination, and zenith angle of the sky patch. The solar radio flux values ("Observed, Series C") are provided by the Dominion Radio Astrophysical Observatory (DRAO). They correspond to the day prior to date of the given sky measurement.

Including the data given in Paper I, we have 84 usable values, on 79 nights, of the $V$-band night-sky brightness in our data base. This amounts to 78 values on 75 nights at Hale Pohaku, the rest having been obtained at sea level or at the Mauna Kea summit. In Paper I we found no discernible difference in the zenith night-sky brightness obtained at these three light-pollution-free locations. However, because of the increasing number of lights at Hale Pohaku over the years, even though the street lights in the parking lots are shielded, the night-sky brightness at Hale Pohaku must now be undoubtedly a bit brighter than it is at the summit. Our data base also contains 70 usable $B$-band measures on 67 nights. The data are termed "usable" if obtained on clear moonless nights, and at galactic latitude $|b|>10^{\circ}$. This excludes data taken when the Moon was just below the horizon.

ET

Table 2 gives the observed yearly averages of sky brightness obtained from 1985 through 1996. We can obtain an estimate of the internal error of an individual measurement by subtracting off the yearly averages from the data and computing the Gaussian standard deviation of the resultant distribution. For the $V$-band measures we obtain $\sigma_{V}= \pm 0.173$ $\mu_{V}$, and for the $B$-band measures we obtain $\sigma_{B}= \pm 0.181 \mu_{B}$. The mean color of the sky is $<B-V>=0.930 \pm 0.018$ based on 70 points.

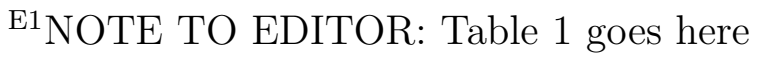


$\mathrm{E2}$

Our data exhibit no statistically significant variations of sky color over the course of the sunspot cycle. Perhaps there $i s$ a variation, but given the internal error of an individual sky color $( \pm 0.147 \mathrm{mag})$, it might take a century to demonstrate any color variation, or at least many more points per year over the course of one whole solar cycle. As a rule of thumb we can state that one need not necessarily measure the $B$-band sky brightness on a given occasion to have the value, if one has measured the stronger signal due to the $V$-band sky brightness.

In Fig. 1 we plot the the values from Table 2. For comparison we plot in Fig. 2 the monthly averages of the $10.7 \mathrm{~cm}$ solar flux obtained from DRAO.

\section{Discussion}

Because magnitudes are a logarithmic unit, for further analysis of the $V$-band measures we need to convert the values of $\mathrm{I}\left(\mu_{V}\right)$ to some linear brightness unit. In times past one used the unusual unit $\mathrm{S}_{10}(V)$, the number of 10th magnitude stars per square degree. More recent studies (e.g. Garstang 1989, Schaefer 1990) have settled on the nanoLambert (nL). Allen (1973, p. 26) states that the surface brightness equivalent of one $\mathrm{m}_{V}=0$ star per square degree is $2.63 \times 10^{-6}$ Lamberts. Since a 10 th magnitude star is exactly $10^{4}$ times fainter than a 0 magnitude star, $\mathrm{S}_{10}(\mathrm{~V})=1$ is the same as $0.263 \mathrm{~nL}$. Let $a \equiv(100)^{0.2} \approx 2.51189$, and let $Q \equiv 10.0+2.5 \log \left(3600^{2}\right) \approx 27.78151$. Q would be the brightness of a single square arc second of sky if a $V=10$ star were spread out over one square degree and that were the only source of light. The observed sky brightness, $\mathrm{B}_{o b s}$, in $\mathrm{nL}$ is then related to the $V$-band sky brightness, $\mathrm{I}\left(\mu_{V}\right)$, in magnitudes per square arc second, as follows:

\footnotetext{
${ }^{\text {E2} N O T E ~ T O ~ E D I T O R: ~ T a b l e ~} 2$ goes here
} 


$$
B_{\text {obs }}(n L)=0.263 a^{\left[Q-I\left(\mu_{V}\right)\right]} .
$$

It is also possible (Garstang 1989, Equation 28), to relate the $V$-band sky brightness in magnitudes per square arc second to the brightness $b_{v}$ of the sky, expressed in photons $\mathrm{cm}^{-2} \mathrm{sec}^{-1}$ steradian $^{-1}$, as follows:

$$
I\left(\mu_{V}\right)=41.438-1.0857 \ln \left(\mathrm{b}_{\mathrm{V}}\right) .
$$

For the $B$-band the first coefficient is changed to 41.965 (Garstang 1989, Equation 39). Future work might sensibly use photon units, since nL are strictly applicable only to the eye, only approximately valid for the $V$-band, and not applicable to $B$-band measures.

The sky brightness is faintest at the zenith. We use Equation 19 of Schaefer (1990) to correct the observed sky brightness to the zenith value:

$$
B_{z e n}=B_{o b s} /\left(1+Z^{2} / 2\right)
$$

where $\mathrm{Z}$ is the zenith angle of the sky patch in radians. A more elaborate relation (i.e. Equation 7 below) must be used for large zenith angles.

In Fig. 3 we give the yearly averages of sky brightness converted to $\mathrm{nL}$ as a function of the $10.7 \mathrm{~cm}$ solar flux on the dates when sky brightness was measured. We cannot account for the anomalous yearly average of 1985. Perhaps we were not careful enough to exclude visible stars from the sky patch. Perhaps there was extra light scattered off of aerosols or dust from the ongoing series of eruptions of Kilauea Volcano on the Big Island. These eruptions began 3 January 1983. The anomalous point of 1993, however, is probably due to ash from the Philippine volcano Mt. Pinatubo. This ash reached Hawaii on 1 July 1991, just in time to degrade the sky for our solar eclipse of 11 July. It gave us yellowish-grey 
sky during the day for nearly two years, colored sunsets, and increased optical wavelength extinction values at least through the spring of 1993. However, what is suspect about our hypothesis for the 1993 point in Fig. 3 is that the data of the second half of 1991 and for 1992 are not above the line. The highest point in Fig. 3, at $\mathrm{B}_{z e n}=96.5 \mathrm{~nL}$, is for the year 1990.

The least-squares line, fit to the dots in Fig. 3 and weighted by the errors of the points, is:

$$
B_{z e n}=(47.021 \pm 2.784)+(0.2019 \pm 0.0222) \times(10.7 \mathrm{~cm} \text { solar flux })
$$

From 1947 to 1988 the observed $10.7 \mathrm{~cm}$ solar flux ranged from 63.0 to $383.4 \times 10^{-22}$ $\mathrm{W} / \mathrm{m}^{2} / \mathrm{Hz}$ (Walker 1988). The data of 1989 through 1996 do not appear to have exceeded these extrema. Taking the extrema of the observed daily solar flux, the implied range of $\mathrm{B}_{z e n}$ is 59.74 to $124.43 \mathrm{~nL}$, or $21.89>\mathrm{I}\left(\mu_{V}\right)>21.09$. The corresponding $B$-band range would be $22.82>\mathrm{I}\left(\mu_{B}\right)>22.02$. One would expect this range of sky brightness at any of the world's choice astronomical sites.

Given our yearly means of $V$-band sky brightness, corrected to the zenith, which ranged from 58.0 to $96.5 \mathrm{~nL}$, Equation 5 implies that the airglow contribution ranged from 11.0 to $49.5 \mathrm{~nL}$, a factor of 4.5. Equation 5 also implies that without the airglow we would detect $47.0 \pm 2.8 \mathrm{~nL}$ due to zodiacal light, faint stars in the beam, and diffuse extragalactic light. We can estimate that $49.4 \mathrm{~S}_{10}(\mathrm{~V})=13.0 \mathrm{~nL}$ is due to stars $V=13$ and fainter (Allen 1973, p. 245) - stars we could not avoid because they are not visible in the eyepiece of our simple photometer with a 15-cm telescope. Tyson (1995) indicates that the diffuse extragalactic light amounts to $0.53 \mathrm{~S}_{10}(\mathrm{~V})$, or $0.14 \mathrm{~nL}$. Thus, roughly, we would estimate that the average amount of zodiacal light we measured was 33.9 ( \pm 3 or more) nL, the 
equivalent of $129 \pm 11 \mathrm{~S}_{10}(\mathrm{~V})$. Since our average sky patch was taken at the zenith $3 \mathrm{~h} 34 \mathrm{~m}$ after the end of twilight at latitude $+20^{\circ}$, this corresponds to an average ecliptic longitude $157^{\circ}$ east of the Sun. The ecliptic latitude of the zenith at Mauna Kea is $-3^{\circ}<\beta<43^{\circ}$, depending on the time of night and the day of the year. Roach \& Gordon (1973, p. 46) indicate that the zodiacal light contribution at $\lambda-\lambda_{\odot} \approx 157^{\circ}$ ranges from $171 \mathrm{~S}_{10}(\mathrm{~V})$ on the ecliptic to $105 \mathrm{~S}_{10}(\mathrm{~V})$ at $\beta=43^{\circ}$. The value at the mid-range, $\beta \approx 22^{\circ}$, is about 133 $\mathrm{S}_{10}(\mathrm{~V})$, in excellent agreement with our value just given. This gives us further confidence that we have no serious source of systematic error in the calibration of our sky brightness values. (We do note, however, that Levasseur-Regourd \& Dumont (1980) found $108 \mathrm{~S}_{10}(\mathrm{~V})$ for the zodiacal light at our mean ecliptic coordinates.)

Garstang's (1989) Equations 28 and 39 let us relate the color in magnitudes (or in magnitudes per square arc second, $\mathrm{I}(\mu))$ of some night sky component to the photon fluxes in photons $\mathrm{cm}^{-2} \mathrm{sec}^{-1}$ steradian $^{-1}$, as follows:

$$
I\left(\mu_{B}\right)-I\left(\mu_{V}\right)=0.527-1.0857 \ln \left(\frac{\mathrm{b}_{\mathrm{B}}}{\mathrm{b}_{\mathrm{V}}}\right)
$$

For the total light of the night sky we find $B-V=0.930$, so the $B$-band to $V$-band flux ratio is 0.690 . For the zodiacal light only, which should be the color of the Sun, $B-V=0.65$, the photon ratio would be 0.893 . This allows us to determine the $B$-band contribution of the zodiacal light. Assuming that the photographic star counts (Allen 1973, p. 245) are equal to the $B$-band star counts, we can then derive the $B$-band flux due to the airglow only. The $V$-band airglow, which is due to continuum plus $557.7 \mathrm{~nm}[\mathrm{O} \mathrm{I}]$ emission, ranges from $1.2 \times 10^{7}$ (solar minimum) to $5.5 \times 10^{7}$ photons $\mathrm{cm}^{-2} \mathrm{sec}^{-1}$ steradian $^{-1}$ (solar maximum). The $B$-band airglow, which is essentially all continuum, ranges from $<0.6 \times$ $10^{7}$ photons $\mathrm{cm}^{-2} \mathrm{sec}^{-1}$ steradian $^{-1}$ at solar minimum to $2.8 \times 10^{7}$ at solar maximum. The $V$-band airglow intensity therefore increases by roughly twice the $B$-band increase $(4.3 \times$ $10^{7}$ vs. $>2.2 \times 10^{7}$ photons $\mathrm{cm}^{-2} \mathrm{sec}^{-1}$ steradian $^{-1}$ ) over the course of the solar cycle. The 
constancy of color provides a possibly useful restriction on the correlations between the airglow $V$ and $B$ intensities.

Walker (1988) found that the sky brightness on many nights decreased by $0.4 \mu$ in the six hours after the end of astronomical twilight. To investigate whether our data corroborates this, we subtracted the individual sky brightness values (converted to nL and corrected to the zenith) from the regression line given by Equation 5. The data set as a whole just gives a scattergram. However, if we restrict ourselves to a subset of the data (1 September 1986 to 3 July 1987 and 20 December 1993 to 16 August 1996), which were obtained during solar minimum, we find marginal support for Walker's finding, which we show in Fig. 4. Excluding the low point of 18 June 1995 from the least-squares fit, we find

$$
\Delta B_{z e n}(n L)=(10.07 \pm 3.24)-(1.79 \pm 0.74) \times \Delta T_{t w i},
$$

where $\Delta \mathrm{T}_{t w i}$ is measured in decimal hours.

The least-squares slope in Fig. 4, which excludes one of the points, is only non-zero at the $2.4-\sigma$ level. The implied nightly trend would be a diminution of the sky brightness by $10.76 \mathrm{~nL}$ over the six hours since the end of twilight. Given the average of the individual data points (corrected to the zenith) that went into Fig. 4, this implies a sky brightness change from 69.63 to $58.87 \mathrm{~nL}$, or $\mathrm{I}=21.72 \rightarrow 21.91 \mu_{V}$, about half the change found by Walker.

Let us now consider the sources of uncertainty in a single sky brightness measurement. First, the sources of systematic error:

(1) Beam area. This affects every derived value of sky brightness the same way (see Equation 2). Our $1-\sigma$ uncertainty contributes $\pm 0.031 \mu$ to the sky brightness values.

(2) Gain difference of amplifier ( $\Delta \mathrm{G}$ in Equation 1). This uncertainty is zero if the sky is 
observed on the same amplifier gain setting as the standard star, and is probably less than $0.01 \mathrm{mag}$ for the most common gain settings used on stars $\left(10^{5.0}\right.$ and $\left.10^{5.5}\right)$.

(3) Gain difference of strip chart settings. We assume this to be negligible for the Hewlett Packard unit that we used, but it is not necessarily zero.

(4) The average amount of faint background stars in the beam. We assume that stars fainter than $\mathrm{V}=19$ would be very difficult for anyone with a reasonably large beam to avoid. We can estimate from Allen (1973, p. 245) that $V=13$ to 19 stars contribute 44.9 $\mathrm{S}_{10}(\mathrm{~V})$ units, or $11.81 \mathrm{~nL}$ (on average), to the sky brightness. If we could have avoided these stars altogether by using a much more sophisticated telescope, a smaller beam and locations carefully chosen from the Palomar Observatory Sky Survey, our brightest observed yearly average (not corrected to the zenith) of $21.287 \mu_{V}$, would have been $21.42 \mu_{V}$, and the faintest observed yearly average of $21.906 \mu_{V}$ would have been $22.15 \mu_{V}$. Choosing sky patches devoid of stars was the method used by Mattila et al. (1996) at La Silla, but their values still exhibit quite a lot of scatter, indicating that the sources of random error (6) and/or (7) below are very significant.

Now let us consider the sources of random error:

(1) Calculating the air mass value of the standard star. For objects close to the horizon, calculating the path length of atmosphere along the line of sight is non-trivial, but within 60 degrees of the zenith the air mass is well approximated by the secant of the zenith angle. For our purposes here, except for calculational errors (using the wrong coordinates of the star or site), this is not a source of uncertainty. [3

(2) Uncertainty of the catalog magnitude of the standard star. Typically \pm 0.01 mag.

\footnotetext{
${ }^{3}$ We mention calculational errors because of examples such as the "first pulsar planet" (Lyne \& Bailes 1992), which resulted from insufficiently accurate coordinates of the object.
} 
(3) Digitizing the signal of the standard star. On average \pm 0.02 mag.

(4) Uncertainty in the adopted extinction. From extinction values given in Paper I, the internal error of the $V$-band extinction at Hale Pohaku is estimated to be \pm 0.03 magnitudes per air mass on nights when the extinction is actually measured, and \pm 0.06 if we just estimate it from the long term seasonal averages and a subjective estimate of the quality of the night. Since the observations (of 1987 to 1996) were made at a median zenith angle of 15.4 degrees (for which air mass $\mathrm{X}=1.037$ ) with a standard star close by on the sky, the uncertainty in the next to last term of Equation 1 is essentially the uncertainty in the extinction.

(5) Digitizing the sky brightness reading. Certainly \pm 0.05 mag, probably more.

(6) The "time-since-the-end-of-twilight" effect found by Walker (1988). The average uncertainty due to this effect at Mauna Kea is unknown, since we only find it marginally at some times near solar minimum.

(7) Variations of sky brightness over the course of minutes (see Fig. 4 of Pilachowski et al. 1989, Fig. 3 of Nather et al. 1990, Morrison et al. 1997, and the discussion below). Nather (private communication) indicates that this can amount to a 100 percent change over the course of an hour.

(8) Differing amounts of faint background stars in the beam. If the faint star background varies by 50 percent of its average value, or $\pm 6 \mathrm{~nL}$, it would translate to $\pm 0.063 \mu_{V}$ at $21.3 \mu_{V}$, and to $\pm 0.110 \mu_{V}$ at $21.9 \mu_{V}$.

The quadratic sum of the first four real sources of random error $(0.01,0.02,0.06$, and 0.05$)$ would give an internal error of $\pm 0.081 \mathrm{mag}$, which is to be compared with our observed internal error of a given $V$-band sky brightness value of $\pm 0.173 \mu_{V}$. Even if (5) above is $\pm 0.10 \mathrm{mag}$, the sources of random error (6), (7), and (8) above must contribute 
over half of the variance. Their contribution to the internal error of a single reading must amount to at least \pm 0.13 mag.

Morrison et al. (1997) and Morrison (private communication) discuss short term variations in the $R$-band. They find 50 percent changes in their photometric zero point on time scales of minutes and even find sky brightness changes over very small angular scales (i.e. different parts of the same CCD frame). It is becoming clear that area photometry at the highest levels of precision must take into consideration what the airglow variations really are - low-level auroral activity.

To investigate further these short term changes of sky brightness, we obtained some data from the Whole Earth Telescope project. This amounts to three-channel photometry (program star, check star, sky) in white light (i.e. with no filter). The data are from two observing runs at the Canada-France-Hawaii Telescope at Mauna Kea that ran from 31 March to 3 April and 20 to 24 May 1991 UT. (This was during the last solar maximum.) During both of these observing runs the Moon was waxing, so it was above the horizon in the evening, and set before dawn.

The WET data amount to thousands of 10 second integrations. The dark count was less than 10 per second. (Given that the count rate on the sky amounted to thousands of counts per second, we can ignore the dark count.) Given that the data are white light counts, not in any photometric band, it would be difficult to calibrate the WET sky brightness counts to values in nanoLamberts using observations of the stars. We can use the net measurements on the check stars to determine the atmospheric extinction for the white light observations. (Given the quantum efficiency as a function of wavelength of the blue sensitive photomultiplier tubes, we expected, and found, extinctions comparable to those one would measure at Mauna Kea in the $U$ - or $B$-bands.)

From the check star measures we could determine when the sky was photometric, and 
could determine a value of the extinction each night. For our purposes here we needed to eliminate the data taken when the Moon was adding to the sky counts. For crescent and quarter Moons, one may include data obtained when the Moon's zenith angle is greater than $94^{\circ}$. For a gibbous Moon the critical zenith angle is about $96^{\circ}$. (It would likely be a still greater value for full Moon.)

Because some of the data were taken at high air mass (3.1), to derive the zenith counts from the observed counts one must do something different than is done above with Equation 4. Instead, it is more appropriate to use

$$
B_{z e n}=B_{o b s} 10^{+0.4 k(X-1)} / X
$$

where

$$
X=\left(1-0.96 \sin ^{2} \mathrm{Z}\right)^{-0.5}
$$

is the optical path length along a line of sight in units of air masses (not quite the secant of the zenith angle), $k$ is the atmospheric extinction in magnitudes per air mass, and $\mathrm{Z}$ is the zenith angle. Equations 7 and 8 are given by Krisciunas \& Schaefer (1991) and are based on the ideas of Garstang (1989).

In Fig. 5 we show the sky counts (reduced to the zenith) from the WET data files on those portions of six photometric nights when the moon was sufficiently below the horizon. We cut off the data for each night 5 minutes before the onset of morning astronomical twilight (defined to be when the Sun elevation angle is $-18^{\circ}$ ). Note that for plotting purposes the data for given nights have been offset. Of particular interest is the night of 31 March 1990. The first part of the data on that night was taken from 1.2 to 3.1 air masses, then a new field at 1.3 air masses was observed until the end of the night. Given that the 
two sets of data on that night knit together well, we have confidence that the sky was photometric, we used an appropriate value of atmospheric extinction, and that Equations 7 and 8 above are appropriate for sky brightness measures that extend to high air masses.

Let us consider the relative change of the sky brightness by defining it as the slope of one of the linear portions of one of the subsections of data in Fig. 5 divided by the mean sky count for that subsection. We find that the zenith sky counts often increase or decrease 5 to 8 percent per hour, but the rate of change can be as high as 22 percent per hour. If we take the logarithms to the base 10 of the sky counts and then multiply them by 2.5 , the corresponding sky brightness change is typically between 0.05 and $0.09 \mathrm{mag} \mathrm{h}^{-1}$, but it can be large as $0.24 \mathrm{mag} \mathrm{h}^{-1}$ (i.e. on the night of 21 May 1991).

By contrast, we found above, from considerations such as those in Fig. 4, a not very convincing sky brightness change of 2.8 percent per hour, or $0.030 \mathrm{mag} \mathrm{h}^{-1}$. Walker (1988) found an exponential, not linear, nightly sky brightness effect which was primarily limited to the first 3 hours after the end of evening astronomical twilight. His average sky brightness change amounted to $0.07 \mathrm{mag} \mathrm{h}^{-1}$. More recently Garstang (1997) has reanalyzed Walker's data, subtracting the estimated contributions due to light pollution, zodiacal light, and faint stars in the beam. This eliminated the sharp curvature during the first two hours of the night, but still showed a slow decline $(\approx 3.7$ percent per hour $)$ with scatter similar to our Fig. 4.

On 3 of the 6 nights shown in Fig. 5 the sky brightness started to increase half an hour to an hour before the start of astronomical twilight. Could this be due to the reverse of Walker's night sky brightness effect, namely, the energization of the upper atmosphere owing the Sun's light from the coming day? ff Or is this just part of the random variations of the sky brightness?

\footnotetext{
${ }^{4}$ If we consider that the typical emission height of the airglow is $90 \mathrm{~km}$ above the surface
} 
Finally, we would wish to know if there is any periodicity in the short term variations of sky brightness. Consider that the Earth has a size (but hardly the mass!) comparable to that of a white dwarf star, and if the variations of the sky brightness are due to gravity-mode oscillations in the Earth's atmosphere, they might occur on time scales comparable (i.e. tens of minutes) to the $g$-mode oscillations of white dwarfs. One might also attempt to correlate sky brightness changes with the twice-daily tides. From power spectrum analysis of the WET sky counts, we find no evidence for any periodicities in the data. However, long stretches of WET data might reveal power at some frequencies, especially at 1 cycle per day if Walker's nightly sky brightness effect regularly takes place.

\section{Conclusions}

We have shown that the zenith $V$-band sky brightness at the mid-level of Mauna Kea varies between 21.3 to 21.9 magnitudes per square arc second over the course of a complete sunspot cycle. Once the solar cycle effect is removed from the data, the largest contribution to the internal scatter of individual measurements of sky brightness would appear to be the short term variations on time scales of tens of minutes. These short term variations have never been studied in detail. Power spectra of some Whole Earth Telescope data presented here indicate that the variations are random, not periodic.

From the data with our system and references given above we find that the zenith $V$-band sky brightness breaks down as follows: (1) airglow, 11.0 to $49.5 \mathrm{~nL}$, varying quite smoothly over the course of the 11-year solar cycle; (2) mean zodiacal light, $33.9 \mathrm{~nL} ;(3)$

\footnotetext{
of the Earth (Roach \& Gordon 1973, p. 54), this seems unlikely. It would require "currents" from the morning terminator to be moving west at roughly twice the rotational speed of the Earth.
} 
mean background stars, $13.0 \mathrm{~nL}$; and (4) extragalactic background light, $0.14 \mathrm{~nL}$. (To convert these numbers to units of $\mathrm{S}_{10}(\mathrm{~V})$, divide by 0.263 .) For comparison, a 4 day old Moon at $60^{\circ}$ zenith angle would contribute $29 \mathrm{~nL}$ to the zenith sky brightness, while a quarter Moon would contribute $99 \mathrm{~nL}$ (Krisciunas \& Schaefer 1991, Table 2). (This assumes a $V$-band extinction of 0.172 magnitudes per air mass.) With telescopes that can be accurately set to pre-selected "blank" fields, the background star contribution can be largely eliminated from one's measures.

In spite of these variations of sky brightness at the 2800-m level of Mauna Kea, Hale Pohaku is a very dark site. In practical terms what would these observing conditions mean for the amateur astronomer (i.e. observing with the eye as the detector)? At such a location as Hale Pohaku one can easily detect the nearest quasar, 3C $273\left(\mathrm{~m}_{V}=12.8\right)$ with a $15-\mathrm{cm}$ telescope. While Pluto has been closer to the Sun than Neptune these past years, it too was detectable in a 15-cm f/6 reflector. On 19 June and 10 July 1988 I easily found Pluto, at $\mathrm{m}_{v}=13.7$, well within my personal record of $\mathrm{m}_{v}=14.5$ obtained with the AAVSO chart of the field of R Her on 2 June 1986. Due to the solar cycle effect, one cannot reach one's limit in any given year, but suffice it to say that the Onizuka Visitors' Center at Hale Pohaku is one of the premier locations available to amateur astronomers.

Since the optical sky brightness at the Mauna Kea summit is essentially the same as that at Hale Pohaku, the sky brightness at Mauna Kea and at other light-pollution-free sites will vary by a significant amount on an 11-year time scale. Professional astronomers using expensive telescopes must take into account the solar cycle effect when planning their observing runs on the faintest targets of opportunity and judging the efficacy of their state-of-the-art instruments. Simple data, such as those presented in this paper, are important for providing a frame of reference for the ongoing analysis of the quality of astronomical sites, and for ensuring the preservation of the high quality of those sites. 
I would like to thank Don Hall, the Director of the University of Hawaii's Institute for Astronomy, and the late Tom Krieger, former head of Mauna Kea Support Services, for making small telescope observing possible at Hale Pohaku (with electric power and parking lot lights one can turn off). Tony Tyson provided useful insights concerning the extragalactic background light. I thank Ed Nather and Gerald Handler for information relating to the Whole Earth Telescope project, and would particularly like to thank Travis Metcalfe for providing some WET data to work on. Roy Garstang made a number of very constructive suggestions which improved this paper. Heather Morrison provided additional insights. 


\section{REFERENCES}

Allen, C. W. 1973, Astrophysical Quantities (3d ed.; London: Athlone Press)

Garstang, R. H. 1989, PASP, 101, 306

Garstang, R. H. 1997, BAAS, 29, in press

Krisciunas, K. 1990, PASP, 102, 1052 (Paper I)

Krisciunas, K. 1996, Sky and Telescope, 91, no. 2, 91

Krisciunas, K., and Schaefer, B. E., PASP, 103, 1033

Levasseur-Regourd, A. C., and Dumont, R. 1980, A\&A, 84, 277

Lyne, A. G., and Bailes, M. 1992, Nature, 355, 213

Mattila, K., Väisänen, P., and Appen-Schnur, G. F. O. v. 1996, A\&A, 119, 153

Morrison, H. L., Miller, E. D., Harding, P., Stinebring, D. R., and Boroson, T. A. 1997, AJ, 113,2061

Nather, R. E., Winget, D. E., Clemens, J. C., Hansen, C. J., and Hine, B. P. 1990, ApJ, 361,309

Pilachowski, C. A., Africano, J. L., Goodrich, B. D., and Binkert, W. S. 1989, PASP, 101, 707

Roach, F. E., and Gordon, J. L. 1973, The Light of the Night Sky (Dordrecht: Reidel)

Schaefer, B. E. 1990, PASP, 102, 212

Tyson, J. A. 1995, in Extragalactic Background Radiation, D. Calzetti, M. Livio, and P. Madau, eds., Cambridge Univ. Press, 103

Walker, M. F. 1970, PASP, 82, 672 
Walker, M. F. 1988, PASP, 100, 496

This manuscript was prepared with the AAS IATEX macros v4.0. 
Fig. 1. - Yearly averages of night-sky brightness from Table 2 for the $V$-band (top set of points) and the $B$-band (bottom points).

Fig. 2.- Monthly averages of $10.7 \mathrm{~cm}$ solar flux from DRAO.

Fig. 3.- Yearly averages of $V$-band night-sky brightness, 1985 to 1996, converted to units of nanoLamberts, and corrected to the zenith. The abscissa values are the averages of the $10.7 \mathrm{~cm}$ solar flux on those days when the sky brightness values were obtained. The open triangle corresponds to data of 1985 , the open circle to data of 1993 . The linear fit is to the dots, weighted by the errors of those points.

Fig. 4.- The residuals of individual $V$-band data points from the least-squares line of Equation 5, vs. the time since the end of astronomical twilight. Data of 1 September 1986 to 3 July 1987 and data of 20 December 1993 to 16 August 1996 are shown. The datum of 18 June 1995 (open square) is not included in the linear fit.

Fig. 5.- Uncalibrated 10 second white light integrations on the sky with a blue sensitive photomultiplier tube, obtained during two Whole Earth Telescope runs at the CanadaFrance-Hawaii Telescope at Mauna Kea. The data have been corrected to the zenith via Equations 7 and 8. From bottom to top the data sets have been offset by 0, 0, 5000, 8000, 13000, and 13000 counts. The night of 20 May 1991 did have some cirrus; we include the sky counts at those times when the check star counts were a reasonably smooth function of time. 
Table 1. Individual Observations of Night-Sky Brightness

\begin{tabular}{|c|c|c|c|c|c|c|c|c|}
\hline UT date & $\begin{array}{c}\mathrm{UT}_{V} \\
\text { hh:mm }\end{array}$ & $\begin{array}{l}\Delta \mathrm{T}_{t w i} \\
\text { hh:mm }\end{array}$ & $\begin{array}{c}\alpha \\
\mathrm{hh}: \mathrm{mm}\end{array}$ & $\begin{array}{l}\delta \\
\circ\end{array}$ & $\begin{array}{l}\mathrm{Z} \\
\circ\end{array}$ & $\begin{array}{c}\mathrm{V} \\
\mathrm{mag} / \mathrm{sec}^{2}\end{array}$ & $\begin{array}{c}\text { B } \\
\mathrm{mag} / \mathrm{sec}^{2}\end{array}$ & $\begin{array}{l}10.7 \mathrm{~cm} \odot \text { flux } \\
10^{-22} \mathrm{~W} / \mathrm{m}^{2} / \mathrm{Hz}\end{array}$ \\
\hline \multicolumn{9}{|l|}{1990} \\
\hline $21 \mathrm{Mar}$ & $9: 23$ & $3: 37$ & $11: 14$ & 19.0 & 4.4 & 21.489 & 22.322 & 225.7 \\
\hline 28 Mar & 8:09 & $2: 21$ & $10: 25$ & 19.8 & 2.8 & 21.492 & 22.391 & 216.0 \\
\hline 18 Apr & $6: 48$ & $0: 52$ & $9: 28$ & 20.0 & 10.0 & 21.235 & 22.204 & 234.9 \\
\hline $17 \mathrm{Sep}$ & $8: 06$ & $1: 28$ & $21: 10$ & 19.0 & 4.3 & 21.079 & 21.922 & 203.0 \\
\hline $14 \mathrm{Nov}$ & $10: 58$ & $5: 59$ & $5: 07$ & 19.0 & 13.7 & 21.590 & 22.340 & 185.3 \\
\hline \multicolumn{9}{|l|}{1991} \\
\hline 4 Jan & $6: 22$ & 1:09 & $4: 29$ & 19.2 & 22.4 & 21.444 & 22.315 & 175.8 \\
\hline 7 Mar & $8: 10$ & $2: 28$ & 10:00 & 20.0 & 17.3 & 21.428 & 22.423 & 209.9 \\
\hline $4 \mathrm{Apr}$ & $7: 25$ & $1: 34$ & 8:44 & 18.2 & 16.1 & 21.209 & 22.400 & 195.9 \\
\hline 12 May & 9:02 & $2: 53$ & $15: 41$ & 28.9 & 24.9 & 21.588 & 22.641 & 229.8 \\
\hline 9 Sep & $7: 16$ & $1: 29$ & $17: 27$ & 26.1 & 37.1 & 21.665 & 22.841 & 196.1 \\
\hline $15 \mathrm{Sep}$ & $10: 23$ & $4: 43$ & $0: 39$ & 20.5 & 14.7 & 21.247 & 22.300 & 181.3 \\
\hline $30 \mathrm{Sep}$ & $7: 48$ & $2: 23$ & $20: 40$ & 19.5 & 20.1 & 21.484 & 22.543 & 194.7 \\
\hline 13 Oct & $10: 51$ & $5: 37$ & 1:05 & 20.0 & 22.0 & 20.968 & 21.892 & 188.0 \\
\hline $10 \mathrm{Nov}$ & $9: 52$ & $4: 52$ & $3: 44$ & 20.0 & 13.5 & 21.192 & 22.361 & 197.4 \\
\hline 27 Dec & $8: 11$ & 3:01 & $5: 29$ & 12.9 & 30.2 & 21.536 & $\ldots$ & 260.8 \\
\hline 30 Dec & $7: 43$ & $2: 22$ & $4: 30$ & 19.2 & 30.7 & 21.441 & 22.329 & 254.4 \\
\hline \multicolumn{9}{|l|}{1992} \\
\hline 27 Jan & $7: 38$ & $2: 11$ & $4: 28$ & 19.2 & 19.8 & 21.418 & 22.218 & 209.0 \\
\hline 31 Jan & 10:08 & $4: 39$ & $7: 28$ & 27.8 & 15.4 & 21.370 & 22.284 & 280.3 \\
\hline
\end{tabular}


Table 1-Continued

\begin{tabular}{|c|c|c|c|c|c|c|c|c|}
\hline UT date & $\begin{array}{c}\mathrm{UT}_{V} \\
\text { hh:mm }\end{array}$ & $\begin{array}{l}\Delta \mathrm{T}_{t w i} \\
\mathrm{hh}: \mathrm{mm}\end{array}$ & $\begin{array}{c}\alpha \\
\text { hh:mm }\end{array}$ & $\begin{array}{l}\delta \\
\circ\end{array}$ & Z & $\begin{array}{c}\mathrm{V} \\
\mathrm{mag} / \mathrm{sec}^{2}\end{array}$ & $\begin{array}{c}\text { B } \\
\mathrm{mag} / \mathrm{sec}^{2}\end{array}$ & $\begin{array}{l}10.7 \mathrm{~cm} \odot \text { flux } \\
10^{-22} \mathrm{~W} / \mathrm{m}^{2} / \mathrm{Hz}\end{array}$ \\
\hline $3 \mathrm{Feb}$ & $11: 25$ & $5: 55$ & $8: 45$ & 18.7 & 16.6 & 21.082 & 21.944 & 288.3 \\
\hline $9 \mathrm{Feb}^{a}$ & $12: 48$ & $7: 16$ & 10:24 & 33.7 & 22.2 & 21.252 & 22.234 & 225.0 \\
\hline $23 \mathrm{Mar}$ & $7: 45$ & 2:08 & 10:17 & 19.8 & 11.7 & 21.312 & 22.138 & 160.7 \\
\hline $26 \mathrm{Nov}$ & 9:07 & 4:09 & $4: 31$ & 18.5 & 19.1 & 21.287 & 22.191 & 166.8 \\
\hline \multicolumn{9}{|l|}{1993} \\
\hline $25 \mathrm{Jan}$ & $8: 20$ & $2: 53$ & $4: 29$ & 19.5 & 25.4 & 21.571 & 22.688 & 104.8 \\
\hline 19 Feb & $7: 17$ & $1: 40$ & $4: 30$ & 19.4 & 33.4 & 21.537 & 22.361 & 126.0 \\
\hline 24 Mar & 8:06 & $2: 19$ & $8: 45$ & 18.0 & 15.8 & 21.568 & 22.240 & 120.8 \\
\hline 14 Apr & $6: 57$ & 1:03 & $8: 45$ & 18.4 & 19.0 & 21.207 & 22.144 & 97.2 \\
\hline $23 \mathrm{Apr}$ & $6: 51$ & $0: 52$ & $10: 18$ & 20.0 & 4.9 & 21.315 & 22.106 & 117.1 \\
\hline $11 \mathrm{Nov}$ & $8: 48$ & $3: 49$ & $2: 49$ & 29.2 & 16.7 & 21.416 & 22.326 & 90.1 \\
\hline 20 Dec & $10: 23$ & $5: 17$ & $4: 30$ & 19.2 & 21.5 & 21.808 & 22.295 & 87.0 \\
\hline \multicolumn{9}{|l|}{1994} \\
\hline 7 Mar & $7: 12$ & 1:30 & $5: 41$ & 9.3 & 32.7 & 21.612 & 22.280 & 95.5 \\
\hline $8 \mathrm{Apr}$ & 7:07 & $1: 14$ & $10: 24$ & 33.7 & 15.9 & 21.846 & 22.778 & 72.8 \\
\hline \multicolumn{9}{|l|}{1995} \\
\hline 18 Jun & $8: 44$ & $2: 16$ & $17: 39$ & 12.6 & 23.3 & 22.123 & $\cdots$ & 69.9 \\
\hline 25 Jun & $9: 28$ & 3:00 & $17: 36$ & 12.0 & 28.9 & 21.739 & $\cdots$ & 71.1 \\
\hline $3 \mathrm{Jul}$ & $8: 53$ & $2: 24$ & $17: 39$ & 12.6 & 9.3 & 21.726 & 22.574 & 77.7 \\
\hline
\end{tabular}


Table 1-Continued

\begin{tabular}{|c|c|c|c|c|c|c|c|c|}
\hline UT date & $\begin{array}{c}\mathrm{UT}_{V} \\
\text { hh:mm }\end{array}$ & $\begin{array}{l}\Delta \mathrm{T}_{t w i} \\
\text { hh:mm }\end{array}$ & $\begin{array}{c}\alpha \\
\text { hh:mm }\end{array}$ & $\begin{array}{l}\delta \\
\circ\end{array}$ & $\begin{array}{l}\mathrm{Z} \\
\circ\end{array}$ & $\begin{array}{c}\mathrm{V} \\
\mathrm{mag} / \mathrm{sec}^{2}\end{array}$ & $\begin{array}{c}\mathrm{B} \\
\mathrm{mag} / \mathrm{sec}^{2}\end{array}$ & $\begin{array}{l}10.7 \mathrm{~cm} \odot \text { flux } \\
10^{-22} \mathrm{~W} / \mathrm{m}^{2} / \mathrm{Hz}\end{array}$ \\
\hline \multicolumn{9}{|l|}{1996} \\
\hline $15 \mathrm{Feb}$ & 10:42 & $5: 07$ & 10:20 & 19.5 & 4.9 & 21.964 & 22.917 & 69.0 \\
\hline 11 Mar & $8: 31$ & $2: 47$ & 10:20 & 19.5 & 12.6 & 21.742 & 22.735 & 68.9 \\
\hline 21 Mar & $10: 23$ & $4: 37$ & $13: 10$ & 18.0 & 17.1 & 21.871 & $\ldots$ & 69.3 \\
\hline 23 Mar & 10:33 & $4: 46$ & $12: 35$ & 18.4 & 3.9 & 21.951 & 23.141 & 73.8 \\
\hline $16 \mathrm{Aug}$ & $14: 15$ & 8:08 & $0: 57$ & 23.4 & 9.3 & 22.001 & 22.686 & 67.6 \\
\hline
\end{tabular}

${ }^{a}$ Data obtained at the 4205-m Mauna Kea summit with the 0.6-m telescope. All other data were obtained with a 15-cm telescope at the 2800-m level. 
Table 2. Yearly Averages of Night-Sky Brightness

\begin{tabular}{rcrcr}
\hline \hline & & & & \\
Year & $\mathrm{V}$ & $\mathrm{n}_{V}$ & $\mathrm{~B}$ & $\mathrm{n}_{B}$ \\
& $\mathrm{mag} / \mathrm{sec}^{2}$ & & $\mathrm{mag} / \mathrm{sec}^{2}$ & \\
\hline & & & & \\
1985 & $21.507 \pm 0.094$ & 4 & & \\
1986 & $21.769 \pm 0.053$ & 9 & $22.513 \pm 0.025$ & 4 \\
1987 & $21.752 \pm 0.090$ & 7 & $22.803 \pm 0.104$ & 6 \\
1988 & $21.616 \pm 0.062$ & 11 & $22.498 \pm 0.044$ & 11 \\
1989 & $21.471 \pm 0.043$ & 14 & $22.478 \pm 0.049$ & 14 \\
1990 & $21.377 \pm 0.095$ & 5 & $22.236 \pm 0.084$ & 5 \\
1991 & $21.382 \pm 0.062$ & 11 & $22.404 \pm 0.079$ & 10 \\
1992 & $21.287 \pm 0.048$ & 6 & $22.168 \pm 0.049$ & 6 \\
1993 & $21.489 \pm 0.074$ & 7 & $22.309 \pm 0.072$ & 7 \\
1994 & $21.729 \pm 0.117$ & 2 & $22.529 \pm 0.249$ & 2 \\
1995 & $21.863 \pm 0.130$ & 3 & 22.574 & 1 \\
1996 & $21.906 \pm 0.046$ & 5 & $22.870 \pm 0.103$ & 4 \\
& & & & \\
\hline
\end{tabular}

Note. - The night-sky measurements of 1987 to 1996 were made at a median zenith angle of 15.4 degrees. As a result, the values of night-sky brightness at the zenith for those years would be, on average, $0.039 \mu$ fainter than the values in this table. 


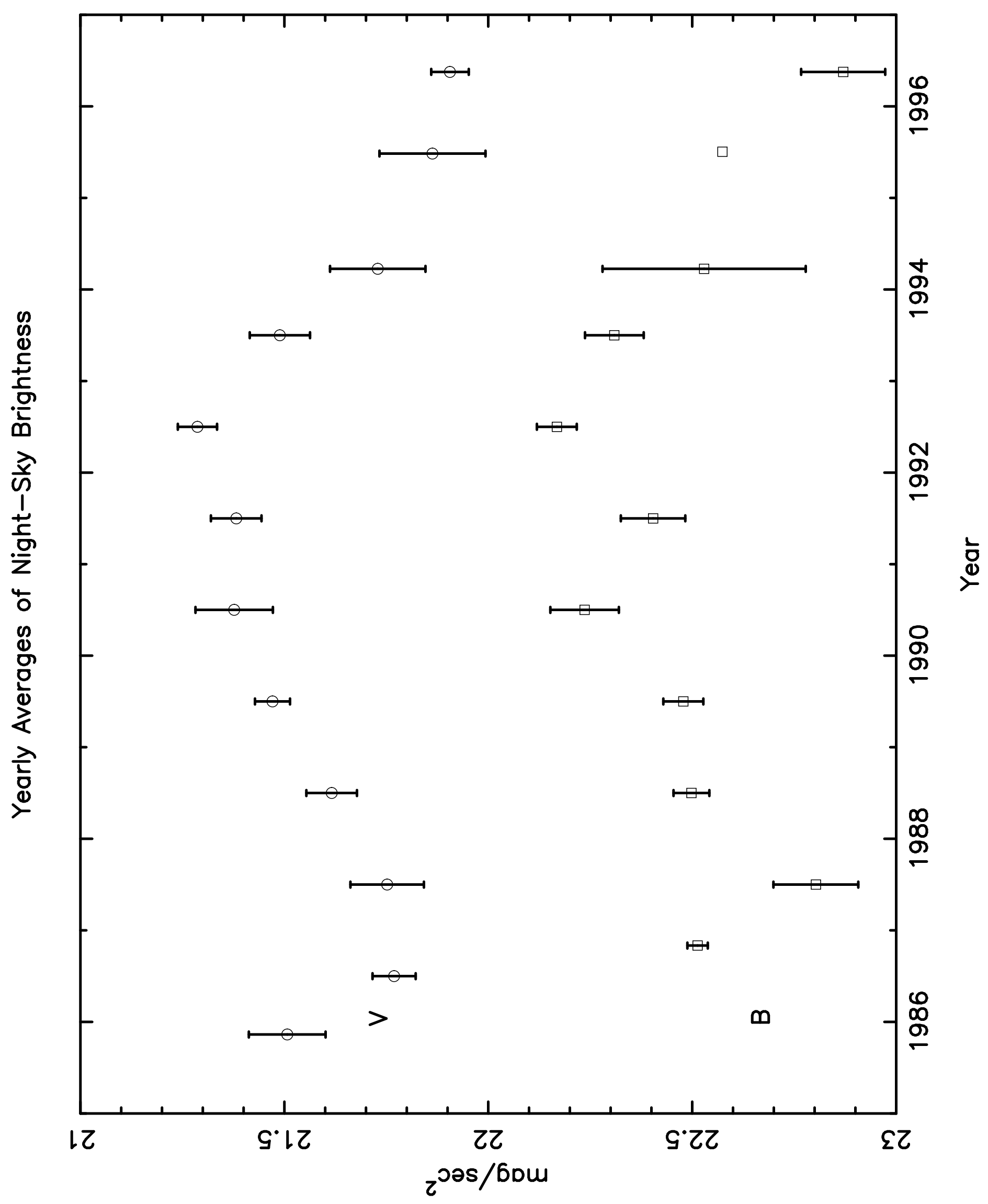




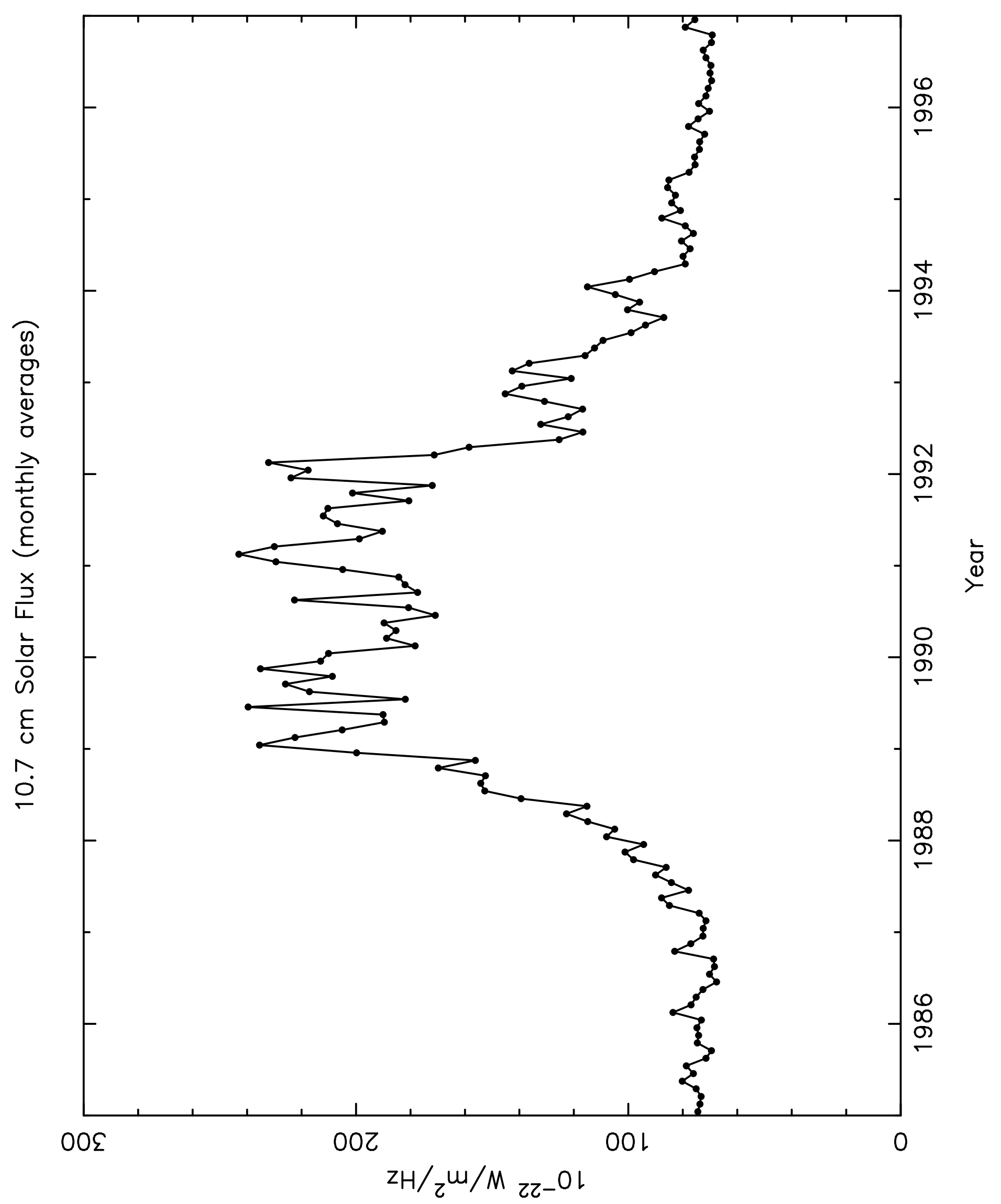




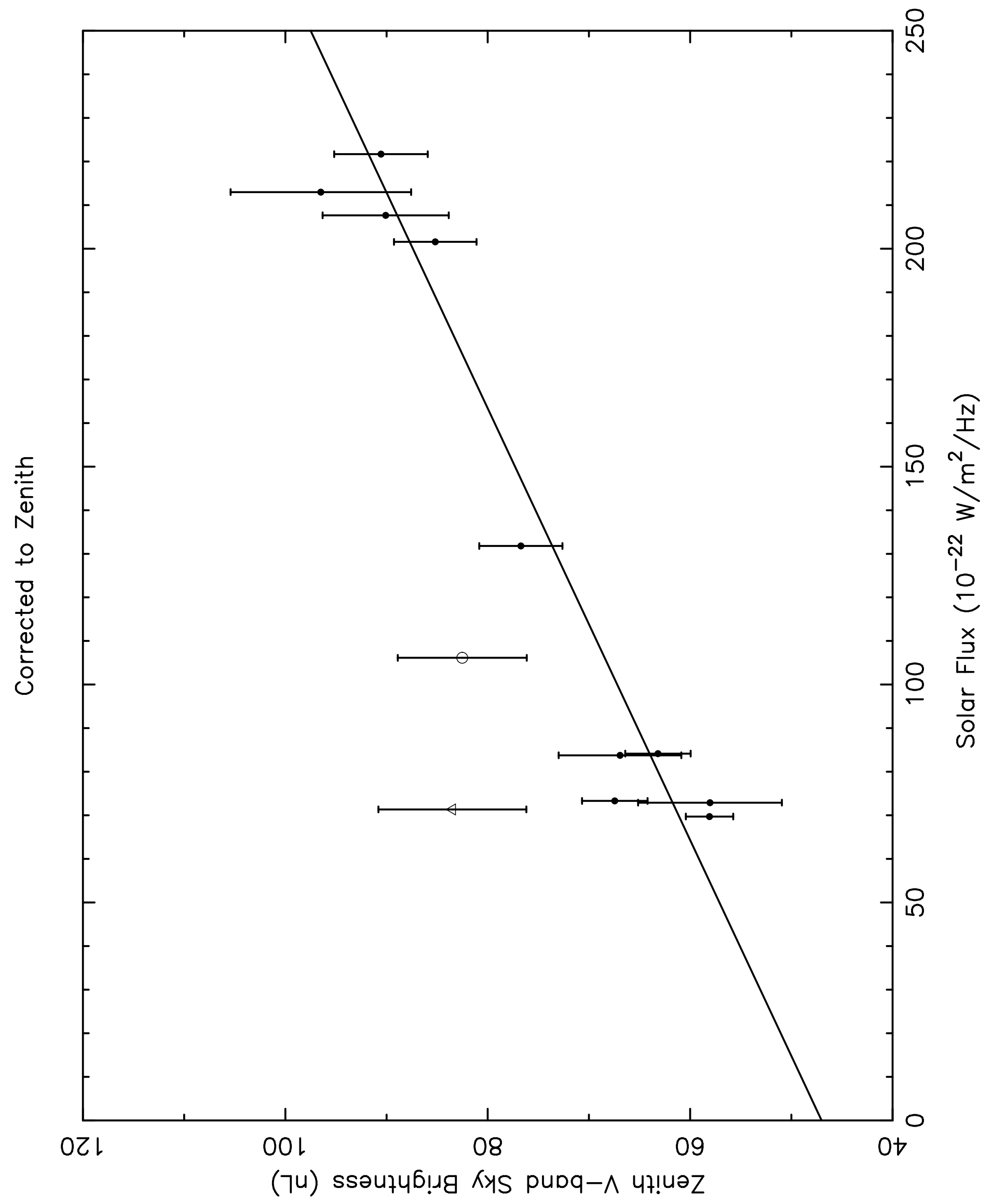




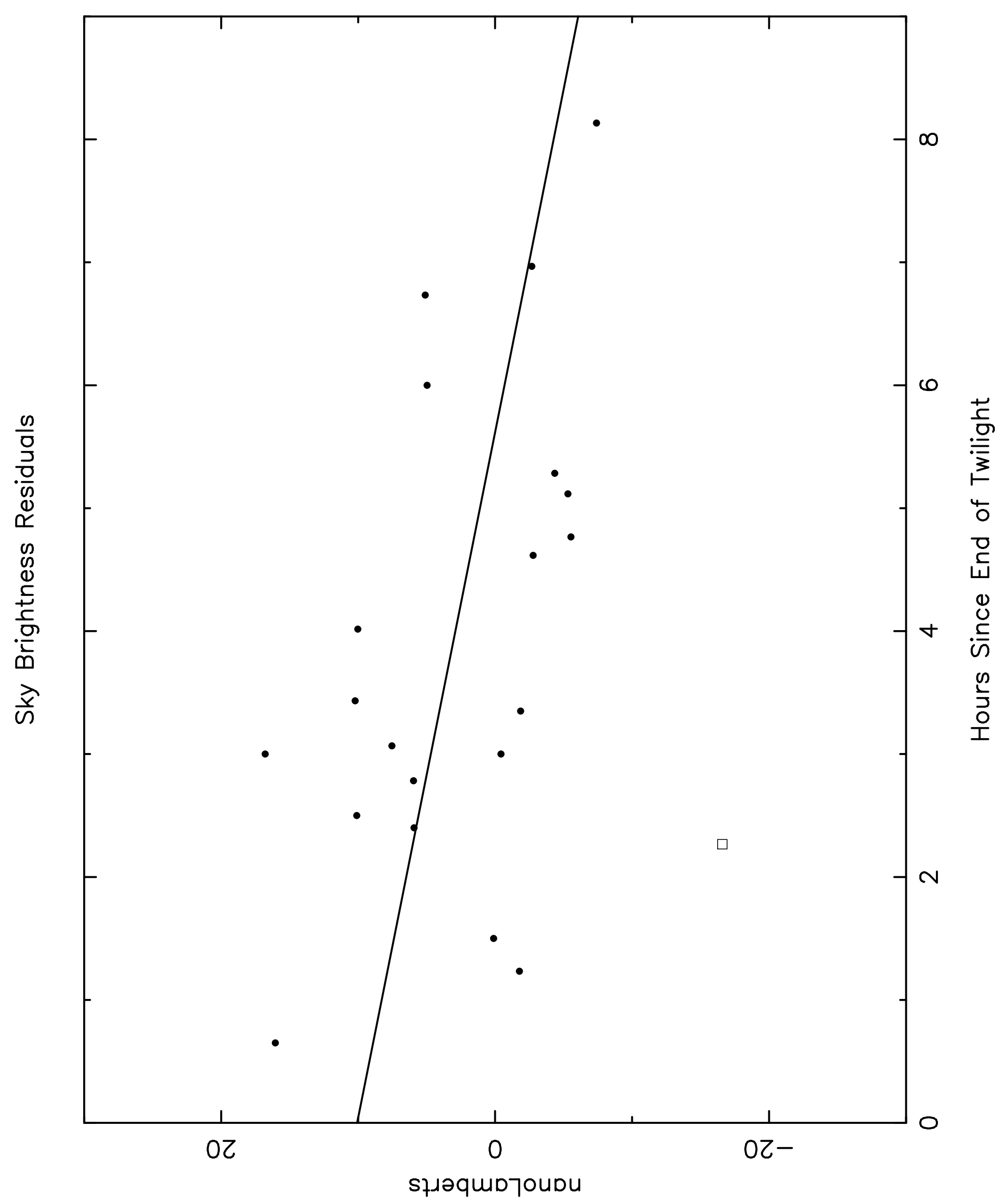




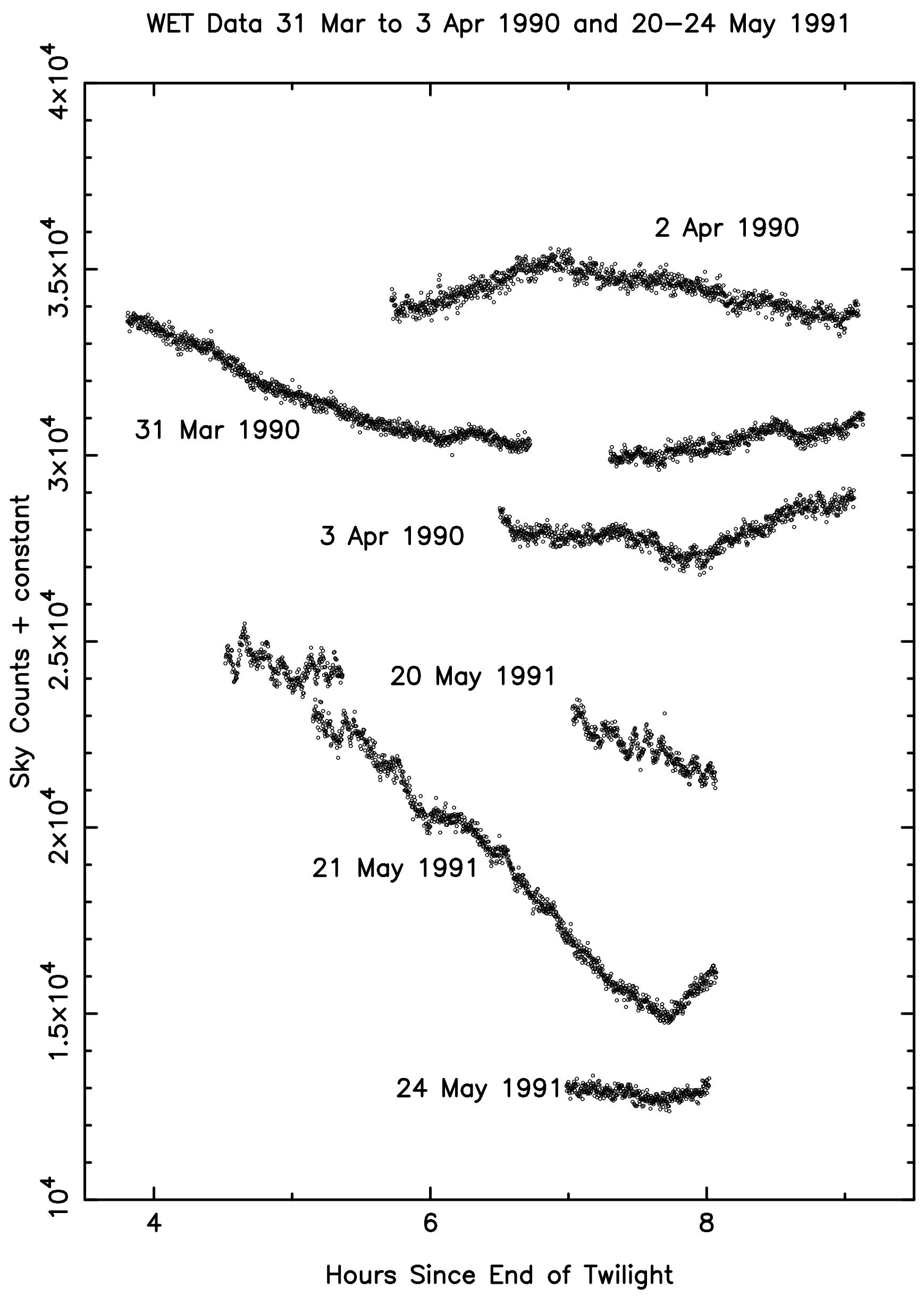

